\title{
NEAR-FIELD COMMUNICATION AND ITS APPLICATIONS - A REVIEW
}

Manjushree $\mathrm{V}^{1}$ and Chethana R Murthy ${ }^{2}$

\begin{abstract}
Near-Field Communication chips may replace every card in the wallet in near future. The potential for NFC stretches beyond commerce. NFC tags can be integrated into smart phones for contactless communication for information transfer, controlling other devices, payment, and many more. NFC is used in variety of applications, making life easier by reducing manual efforts and making job paperless. In this paper we present an overview of NFC technology, NFC enabled smart phone architecture, comparison between NFC and Bluetooth technology, and a review of major NFC applications.
\end{abstract}

Keywords: NFC, RFID, Bluetooth, Applications

\section{INTRODUCTION}

Near-Field Communication (NFC) is based on wireless technology which is now a days replacing wired technology [1]. NFC is a communication technology which operates within a very small proximity range. NFC has a collection of communication protocols that allows two smartphones or devices to communicate with each other by bringing them within 4-10 $\mathrm{cm}$ range. NFC operation is carried out at $13.56 \mathrm{MHZ}$ frequency. Magnetic induction is the major concept that is used for contactless communication. NFC is gaining popular in India because of demonetisation.

\section{BACKGROUND}

NFC is standardized by ISO/14443 - universal Standard for vicinity contactless cards. There are several variants of this standard. Variant 1 describes physical characteristics of NFC smart card. Variant 2 describes the power of radio frequency and signal interface used in the contactless communication. Variant 3 describes the anti-collision properties when two or more tags are in the same proximity limit. Variant 4 describes the protocol used for transmission of contents within the NFC enabled devices.

NFC is based on Radio-Frequency Identification (RFID) technology and uses the same working principles. The key feature of NFC devices is that they can read out RFID transponders and emulate them. Radio frequency identification is a technology that is used while communicating in wireless. Communication to remote NFC tag or device is done by radio frequency interface. This method uses the radio waves to detect the objects that are

\footnotetext{
${ }^{1}$ Department of Information Science \& Engineering $R$ V College of Engineering, Bengaluru, India

${ }^{2}$ Department of Information Science \& Engineering $R V$ College of Engineering, Bengaluru, India
} 
brought into the field. RFID device is made up of a small chip and an antenna. RFID is similar to bar code or magnetic strip on credit card. One of the important advantages of RFID device is it does not use battery, hence the device can be used for longer days [2].

\section{NFC ARCHITECTURE}

More recently NFC chips are stocked inside the smart phones to digitize the entire wallet to access funds for contactless payments. It can also allow transfer of photos, contacts and directions that works by holding two phones together. NFC technology that is embedded in smart phones includes two parts: secure element, host controller and NFC controller. The figure 1 below depicts the typical NFC architecture of an NFC enabled phone.

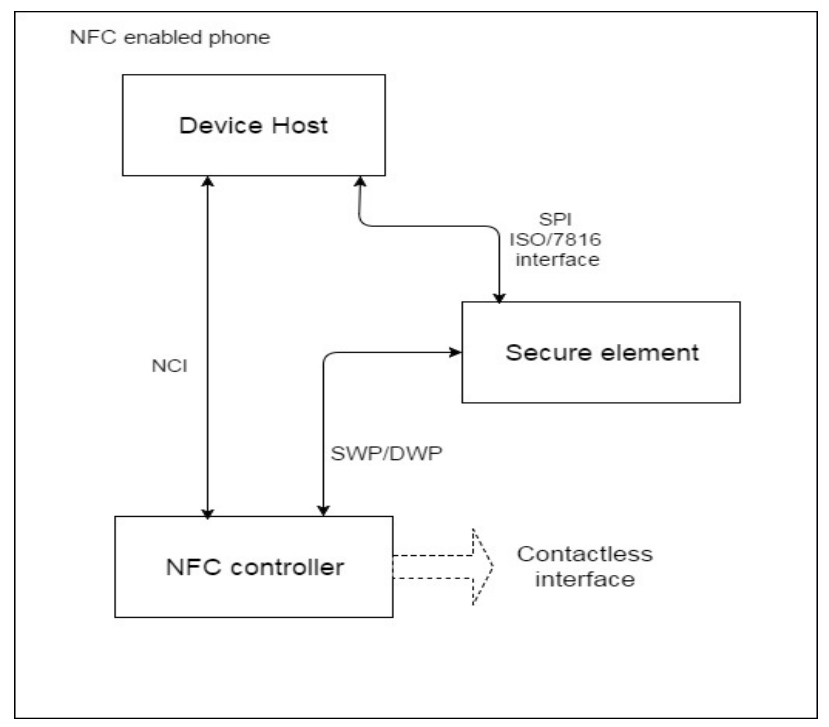

Figure 1. NFC architecture

Secure element can securely host the application and confidential data present on the phone and follows the securing mechanisms involved with NFC as per trusted authority. Since it is contactless communication it must involve more security. Secure element can be accessed internally from the host or externally that the reader connected within the proximity range.

Host controller is an important module of any smart phone. Host controller is the part where middleware resides. Host Controller Interface (HCI) is used for communication between NFC controller and host controller. Host controller processes the data that are sent and received from the user and secure element. It formats data in such a way that secure element can interpret it. The application that supports NFC operation is present in the device host which allows launching and using the application for various operations of NFC.

NFC controller interface acts as interface between device host and NFC controller. For performing secure transactions with NFC, secure element is connected to NFC controller. The interaction between host and the secure element via NFCC use a dual wired protocol which is two-way communication between the two. Various transport mechanisms such as I2C, SPI can be used for communication. This flexibility is provided by the logical interface provide by NFC controller interface. 


\section{COMPARISON OF NFC WITH EXISTING BLUETOOTH TECHNOLOGY}

Bluetooth and NFC both operate on wireless technology. NFC has very low consumption of power as compared with latest Bluetooth versions. Hence NFC can operate within the given power source and no main power is needed. Speed is also a factor to be considered. NFC offers faster transferring of information than Bluetooth with the help of RFID technology used in NFC. Due to inductive coupling used in NFC It usually take less than a second to establish the communication between the two devices.

NFC transmission limit is just $4-10 \mathrm{~cm}$ range. In this case Bluetooth offers a wide range for transmission till 10 meters. Due to the lesser range in NFC it has faster connectivity than Bluetooth since it has lesser range than Bluetooth. NFC operates with only two devices while Bluetooth allows communication with more than one device.

\section{NFC APPLICATIONS}

\subsection{NFC payments}

Credit or Debit card is the payment method that is widely used. The customer may have account in multiple banks and customer has to maintain so many cards. There is also a paper work involved after each transaction to check the amount deduction from the account.

NFC payments are gaining importance in recent days. It just involves tapping within the range. Payment using NFC requires both the smart phone and the sale terminal to be equipped with NFC chip. The applets obtained from other trusted vendors can be used to store the card information which is then used for transactions. The NFC chip embedded in the NFC enabled smart phone allows loading the applets. Applets are specific to the bank and have all the details of the customer account. These applets are applications for NFC which contains necessary payment information. These applications are useful for making payments. Authors in paper [3] present a review on NFC technology and NFC payment application.

\subsection{Ticketing}

Earlier days booking tickets was very time consuming task as people need to stand in queue or book via phone call and the distribution services were also costly. Mobile ticketing was introduced with the use of smartphone. But the major challenge with this was validating the customer.

Mobile ticketing with NFC has increased with increase in smartphone features. Mobile ticketing helps the user to purchase ticket through the smartphone and also helps to validate the customer by tapping the smart phone to the validator. By doing this the secure information and identity of customer is known to the ticket issuer. Mobile ticketing involves steps like registration, provisioning, validation, ticket check. This technique is more echofriendly than paper tickets. Authors in paper [4] detail the problems and solutions of mobile ticketing for transport companies using NFC management.

\subsection{Healthcare}

Health care systems consist of variety of people such as doctors, patients, nurses etc. In health care system maintaining the patient's record is very much important. Maintaining the paperoriented document and carrying them at each visit is tremendous job. Any miscommunication may have a severe impact. 
Use of NFC in health care allows effective communication among patients and other people involved. The accurate data is exchanged between the patients and doctors. This ensures the proper treatment to the patient. This accurate data is also helpful for the pharmacist to know the prescription given by the doctor. NFC is also helpful for self-diagnosis. Electronic data capture system with the help of NFC is used by the patient to assess their health and know the status.

\subsection{Education}

Schools and universities are using NFC technology to move towards smart classes. There is a study presented on assessment of exam papers using NFC technology. Each student instead of writing their identity in the answer paper they are provided with the NFC tags which they attach to the answer scripts. Teacher evaluating the scripts does not know the identity of the student.

NFC technology can be used to improve the student's capability of learning process. This helps students to incorporate the lessons learnt into real life.

\subsection{Physical door access}

In today's growing technology, wireless technology is used to solve many problems faced with lock and keys. The use of physical keys has some disadvantages. Losing the key or duplicating the key may severely affect the security.

Security is an important concern. With NFC door access is highly secure. Keyless access to the home or to a place makes life easier. The concept used here is digital keys. The user is provided with the smart card which has integrated the user identity. When the smart card is brought near the door the user is authenticated and if validated the door will be opened for some time for the person to enter and remains closed. Authors in paper [5] presents a smartphone-based physical access control system where NFC embedded mobile phone is used to authorize the user access by communicating to the central access server. Authors in paper [6] detail on a digital access control system project that uses NFC technology.

\subsection{Attendance}

It is very important for most of the college and universities to maintain the attendance. Attendance is considered before giving grades to students. Maintaining the attendance in the paper and later maintaining the attendance in the college server manually. This is time consuming and requires lot of manual effort.

To overcome these NFC tags are used. Each student is provided with the NFC tag or NFC embedded card. These tags are tapped against the mobile application launched. NFC reader with the use of RFID reads the information from the tag and stores in the server. Server validates the information and updates the attendance details. This reduces the manual work. Authors in paper [7] detail one such system that uses NFC technology and run on mobile as an application.

\subsection{Transit}

The tickets obtained in the paper format for travelling may be lost or there is always insufficient information present. The customers need not to visit to the ticket counter for buying the ticket. 
Mifare a variant of NFC is used for transit applications. It has more security compare to other application. Mifare is mainly designed for transit applications

NFC technology provides access to information anywhere at any time. The travellers can tap their NFC embedded smart phone against the terminal to obtain information about the timings, schedules etc. Tickets are stored virtually and there is less chance of tickets being lost. Updates to the information are easy and can be easily done with wireless technology.

\section{CONCLUSION}

NFC enables two smart phones to communicate by bringing them in short distance. NFC is now present in almost all smart phones which allow faster content transfer. NFC in recent days is replacing the other wireless technologies. NFC is used in many scenarios which helps the people with their daily activities. NFC is used in variety of applications. It makes life easier with the term tapping. This technology reduces lot of manual efforts involved. Security provided with this technology is also an important aspect to be considered.

\section{REFERENCES}

[1] NFC-Forum, Available: http://www.nfc-forum.org

[2] Vedat Coskun,Busra Ozdenizci, and Kerem Ok "The Survey on Near Field Communication”, Sensors 2015, 15, 13348-13405,MDPI.

[3] Nahar Sunny Suresh Shobha, Kajarekar Sunit Pravin Aruna, Manjrekar Devesh Parag Bhagyashree, Kotian Siddhanth Jagdish Sarita "NFC and NFC Payments: A Review" 2016 International Conference on ICT in Business Industry \& Government (ICTBIG), Indore, 2016, pp. 1-7.

[4] U. Biader Ceipidor; C. M. Medaglia; A. Marino; M. Morena; S. Sposato; A. Moroni; P. Di Rollo; M. La Morgia "Mobile ticketing with NFC management for transport companies. Problems and solutions", 5th International Workshop on Near Field Communication (NFC), Zurich, Switzerland, 2013, pp. 1-6.

[5] Christof Arnosti, Dominik Gruntz, Marco Haur, "Secure Physical Access with NFC-enabled Smartphones" IMVS focus report.

[6] Swastika Shukla. Prachi Shroff, Vivek Nair, Rijo Kuruvilla, "Access Management and Control using NFC" International Journal of Science and Research (IJSR), Vol. 5, Issue 3, Mar 2016, pp 564 - 566.

[7] Abhilash Bhise Radhika Khichi, Amol Korde, Prof. Deepali Lokare, "Attendance System Using NFC Technology with Embedded Camera on Mobile Device", International Journal of Advanced Research in Computer and Communication Engineering (IJARCCE), Vol. 4, Issue 2, Feb 2015 February, pp 350 - 353. 